\title{
Overexpression of Glyceraldehyde-3-phospahe Dehydrogenase Is Not Involved in 5-Hydroxytryptamine (5-HT)-Induced Necrosis in Cultured Cerebrocortical Neurons
}

\author{
Hisao Tajima, ${ }^{a}$ Katsuyoshi Sunaga, ${ }^{b}$ Masaharu Tanaka, ${ }^{c}$ Toyoyasu Kuwae, ${ }^{b}$ and Nobuo Katsube ${ }^{*, c}$ \\ ${ }^{a}$ Discovery Research Laboratories I, Minase Institute, Ono Pharmaceutical Co., Ltd.; 3-1-1 Sakurai, Shimamoto, Mishima, \\ Osaka 618-8585, Japan: ${ }^{b}$ Group on Cellular Neurobiology, Josai University; 1-1 Keyakidai, Sakado, Saitama 350-0248, \\ Japan: and ${ }^{c}$ Safety Research Laboratories, Fukui Institute, Ono Pharmaceutical Co. Ltd.; 1-5-2 Yamagishi, Mikuni, \\ Sakai, Fukui 913-8538, Japan. Received March 19, 2004; accepted May 6, 2004
}

Cerebrocortical cell cultures were prepared from 1-d-old rats. On post-culture day 6, 5-hydroxytryptamine (5-HT) was added to the medium and cells were exposed for another $3 \mathrm{~d}$. 5-HT elicited cytotoxicity in a dose-dependent manner, and the survival rate of neuronal cells was decreased to $64.9 \pm 5.0 \%$ at $0.1 \mathrm{~mm}$ concentration. Chromatin staining with Hoechst 33258 and electron microscopy revealed that the 5-HT-induced neuronal death was entirely due to necrosis. Pretreatments with glyceraldehyde-3-phosphate dehydrogenase (GAPDH) antisense oligonucleotide and several classical apoptotic inhibitors did not exhibit neuroprotection in this paradigm. Northern blot analysis showed that the enhancement of GAPDH mRNA levels was undetected during cell death. The present results demonstrate that GAPDH overexpression is not involved in the 5-HT-induced necrotic death pathway.

Key words glyceraldehyde-3-phosphate dehydrogenase; apoptosis; cerebrocortical neurons

The mode of cell death is of particular importance because apoptosis is a normal physiological process that occurs during development to maintain homeostasis in a neuronal population, ${ }^{1)}$ whereas necrosis results in an inflammatory response involving activation of macrophages and/or microglia. ${ }^{2)}$ Furthermore, abnormality of normal apoptosis has been linked to pathogenesis of a number of human diseases including cancer, viral infections, autoimmune diseases and neurodegenerative disorders. ${ }^{3,4)}$ We have reported previously that apoptosis of rat cerebellar ${ }^{5)}$ and cerebral $^{(6)}$ neurons resulting from "aging" of the cultures is strikingly associated with overexpression of a particulate-bound $38-\mathrm{kDa}$ protein, identified subsequently as glyceraldehyde-3-phosphate dehydrogenase (GAPDH; EC 1.2.1.12). Moreover, GAPDH antisense, but not sense, oligodeoxynucleotides specifically suppress the age-induced accumulation of GAPDH mRNA and protein prior to apoptotic neuronal death. ${ }^{5,6)}$ By the same criteria, GAPDH overexpression in the particulate fraction [i.e., nuclear accumulation ${ }^{7,8}$ ] has been implicated in the low $\mathrm{K}^{+}$/serum deprivation-induced cell death of cerebellar neurons. ${ }^{9)}$ However, this death paradigm includes both apoptosis and necrosis, and only the apoptotic component may involve overexpression of GAPDH. GAPDH has also been implicated in certain forms of neurodegenerative diseases. Namely, the products of several genes leading to degenerative brain diseases interact with GAPDH, including huntingtin in Huntington's disease and $\beta$-amyloid precursor protein in Alzheimer's disease. ${ }^{10,11)}$ The current study was undertaken to clarify the restricted implication of GAPDH overexpression in an apoptotic death pathway. The present study directly observed 5-hydroxytryptamine (5-HT) induction of necrotic death in cultured cerebrocortical neurons independent of GAPDH overexpression.

\section{MATERIALS AND METHODS}

Primary Cultures of Rat Cerebrocortical Neurons Cerebrocortical cells (CCCs) were isolated from 1-d-old
Sprague-Dawley rats and cultured as described previously. ${ }^{6}$ Briefly, cerebral cortices dissociated from diencephalic structures and hippocampus were pooled and sliced $(0.4 \mathrm{~mm}$ in thickness) in two orthogonal directions. The cubes were mechanically dispersed in the presence of trypsin $(0.025 \%)$ and DNase $(0.008 \%)$ before being plated onto poly-L-lysinecoated 35-mm culture dishes. Cells were seeded at a density of $0.8 \times 10^{6}$ cells $/ \mathrm{ml}(2 \mathrm{ml} / \mathrm{dish})$ in basal modified Eagle's medium containing $10 \%$ of fetal bovine serum, $25 \mathrm{~mm} \mathrm{KCl}$, $2 \mathrm{~mm}$ glutamine and $100 \mu \mathrm{g} / \mathrm{ml}$ of gentamicin. Cytosine arabinoside $(10 \mu \mathrm{M})$ was added to the culture medium $20 \mathrm{~h}$ after plating to arrest the growth of nonneuronal cells. To assess neuronal survival, cells washed with Locke's solution were double-stained with $0.0008 \%$ fluorescein diacetate (FDA) and $0.0002 \%$ propidium iodide (PI) to discriminate viable and nonviable cells, respectively. ${ }^{5}$ However, moribund CCCs with PI staining readily detached from the growing surface as they were committed to death, $\left.{ }^{6}\right)$ constantly containing approximately 20 cells throughout the assessment of the surviving cells. The surviving cells were therefore measured in terms of the number of FDA-stained positive cells in the photomicrographs of four representative fields $(500 \times 500 \mu \mathrm{m}$ squares containing approximately 100 live cells in the control) from each dish. Cell viability was expressed as a percentage of the $6 \mathrm{~d}$ in vitro (DIV) control. These animal experiments were approved scientifically and ethically by the Pharmacology Graduation Committee at the Josai University and the Animal Experiments Committee at Research Laboratories, Ono Pharmaceutical Co., Ltd.

Electron Microscopic Examination Neurons grown in plastic dishes were respectively pre- and post-fixed with $3 \%$ glutaraldehyde and $1 \% \mathrm{OsO}_{4}$ before dehydration in ethanol and embedment in Quetol 812 (Nisshin EM, Japan) for electron microscopy. In situ embedding of cultures, preparation of ultrathin sections, and double electron staining of those specimens were performed as described previously. ${ }^{5}$ For nucleus staining with Hoechst 33258 (Sigma-Aldrich), CCCs were grown on a cover glass. After removing the culture 
medium, the neurons were washed twice with ice-cold phosphate-buffered saline (PBS), fixed with 3\% glutaraldehyde in PBS for $30 \mathrm{~min}$ at $4{ }^{\circ} \mathrm{C}$ and washed again thrice with PBS. Cells were then stained with Hoechst $33258(0.4 \mu \mathrm{g} / \mathrm{ml}$ in PBS) for $15 \mathrm{~min}$ at $37^{\circ} \mathrm{C}$, washed, mounted in $50 \%$ glycerol in PBS and nuclei were visualized. ${ }^{6}$

Northern Blot Analysis Total RNA isolation and Northern blot analysis were performed essentially as described previously, ${ }^{12)}$ except that the human GAPDH and $\beta$-actin cDNA probe were 1.1- and 1.8-kb in length, respectively (Clontech) and that high-stringency washing of the hybridized blots was performed in $0.1 \times$ standard saline citrate containing $0.1 \%$ SDS at $60^{\circ} \mathrm{C}$ for $10 \mathrm{~min}$. Approximately $9 \mu \mathrm{g}$ of total RNA from each sample was separated by electrophoresis in a $1.2 \%$ agarose-formaldehyde gel. Specific hybridization bands were quantified by charge-coupled device densitometry of the autoradiograms and then normalized to total cellular RNA in each sample.

Synthesis of Antisense and Sense Oligonucleotides The phosphorothioated antisense and sense oligodeoxyribonucleotides against rat GAPDH cDNA were prepared as described previously. ${ }^{5)}$ The antisense oligonucleotide se-

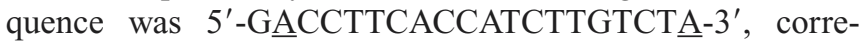
sponding to a sequence flanking the ATG initiation codon (the phosphorothioated nucleotide is underlined). The sequence of sense oligonucleotide was the exact inverse of that of antisense oligonucleotide, with phosphorothioate bonds in the corresponding position.

\section{RESULTS}

Cytotoxicity of 5-HT As in the case of age-induced apoptosis, ${ }^{6)}$ primary cultures of CCCs were prepared from 1d-old rats and cultured in the presence of $25 \mathrm{~mm} \mathrm{KCl}$ without medium change throughout the cultivation period $(9 \mathrm{~d})$ to avoid neuronal death resulting from overexcitation by contaminating glutamate in the fresh medium. ${ }^{13)}$ A single addition of 5-HT at 6 DIV followed by subsequent 3-d exposure induced cell death in this paradigm. A dose-response study on the neurotoxicity of 5-HT revealed that the apparent $\mathrm{LD}_{50}$ value was within the range of $0.3-1.0 \mathrm{~mm}$ in the absence of $10 \mu \mathrm{M}$ pargyline, a monoamine oxidase inhibitor (Fig. 1). This reduction was also reproduced in the presence of $10 \mu \mathrm{M}$ pargyline with a virtually overlapping dose-response (data not shown). 5-HT significantly reduced cell survival to $64.9 \pm 5.0 \%$ of the control at $0.1 \mathrm{~mm}$.

Necrotic Death of Cerebrocortical Cells by 5-HT The 5-HT-induced cell death of CCCs was characterized as necrosis based on the following observations: 1) Chromatin staining with Hoechst 33258 revealed that neurons exposed to 5HT did not display any nuclear fragmentation and/or chromatin condensation (Fig. 2(I)); and 2) Electron microscopic examination of these neurons showed morphological properties typical of necrotic injury; viz., the presence of numerous vacuoles in the cytoplasm with no alteration of nuclear chromatin, and disintegration of the inner mitochondrial membranes (Fig. 2(II)). Furthermore, we detected only a smear pattern of DNA cleavage on agarose gels after electrophoresis of DNA fragments without DNA ladders (multiples of $180 \mathrm{bp}$ ) (data not shown).

The 5-HT-induced neurotoxicity was not affected by the

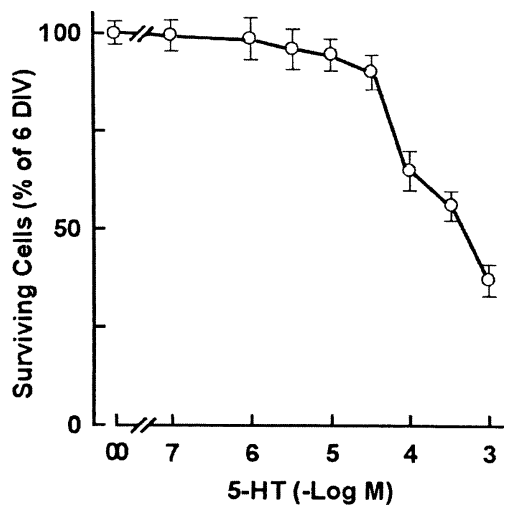

Fig. 1. Dose-Response Relationship for Cytotoxicity of 5-HT on Neuronal Cultures

CCCs were grown in the absence or presence of indicated concentrations of 5-HT for $9 \mathrm{~d}$. When used, the agent was added at 6 DIV and the assessment of cell survival was performed as described in Materials and Methods. Data presented are means \pm S.E.M. of three independent experiments.

addition of cycloheximide (CHX, $0.3 \mu \mathrm{g} / \mathrm{ml})$, a protein synthesis inhibitor; actinomycin-D (Act-D, $0.015 \mu \mathrm{g} / \mathrm{ml}$ ), a RNA synthesis inhibitor; and aurintricarboxylic acid (ATA, $10 \mu \mathrm{M}$ ), an endogenous DNase inhibitor (Fig. 3). Although pretreatments with GAPDH antisense/sense oligonucleotides $(10 \mu \mathrm{M})$ and ketanserin (an antagonist of $5-\mathrm{HT}_{2}$ receptor, $10 \mu \mathrm{M})$ were similarly ineffective, tetrahydroaminoacridine (THA, an antidementia drug, $10 \mu \mathrm{M}$ ) remarkably prevented this neuronal death, scoring an $\mathrm{ED}_{50}$ value of $2.5 \pm 0.2 \mu \mathrm{M}$. Furthermore, MK-801, an antagonist of $N$-methyl-D-aspartate (NMDA) receptor, and several other $5-\mathrm{HT}_{1 \mathrm{C}}$ receptor antagonists, such as mesulergine and ritanserin, did not afford any neuroprotective effect within the tested concentrationrange of $10^{-7}$ to $10^{-4} \mathrm{M}$ (data not shown).

No Changes in Expression Levels of GAPDH mRNA The level of GAPDH mRNA was measured by Northern blot hybridization (Fig. 4A). As in the case of $\beta$-actin mRNA, the level of GAPDH mRNA remained relatively constant at $7-8$ DIV and slight decrease in its level at 9 DIV during necrotic death pathway of CCCs (Fig. 4B). Indeed, the presence of GAPDH antisense oligonucleotide did not affect mRNA levels during the time course studied. In addition, Western blotting for GAPDH protein in this neuronal culture failed to achieve because moribund cells readily detached from the growing surface. ${ }^{6}$

\section{DISCUSSION}

Since 5-HT is a major platelet component, which may be released in large quantities at the site of thrombus formation, it is plausible that locally released 5-HT may be involved in neuronal injury. ${ }^{14)}$ Furthermore, 5-HT suppresses CNS functions in vivo when the blood-brain barrier is disrupted, as may happen at the site of a cerebral infarction. ${ }^{15)}$ Joseph et $a l$. have reported that a relatively high concentration of 5-HT (ca. $350 \mu \mathrm{M})$ induces neurotoxicity in organotypic explant cultures of rat spinal cord, and propose that the findings could provide new insight into the mechanism of cerebral ischemic injury. ${ }^{16)}$ From these aspects, 5-HT was employed as a death-trigger in the present neuronal death model system using cultured rat cerebral neurons.

The present findings demonstrated that long-term adminis- 
I
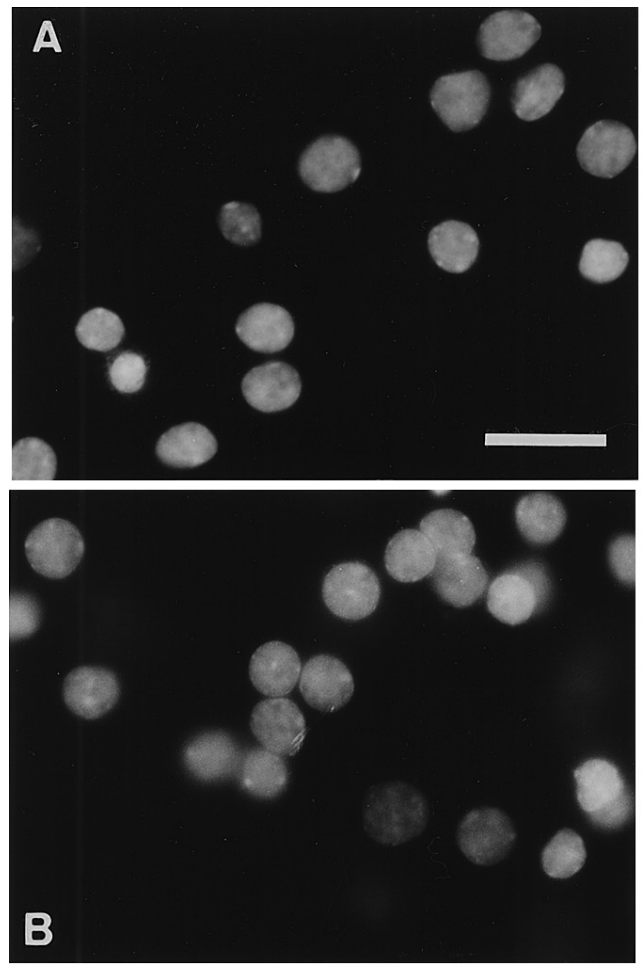

II
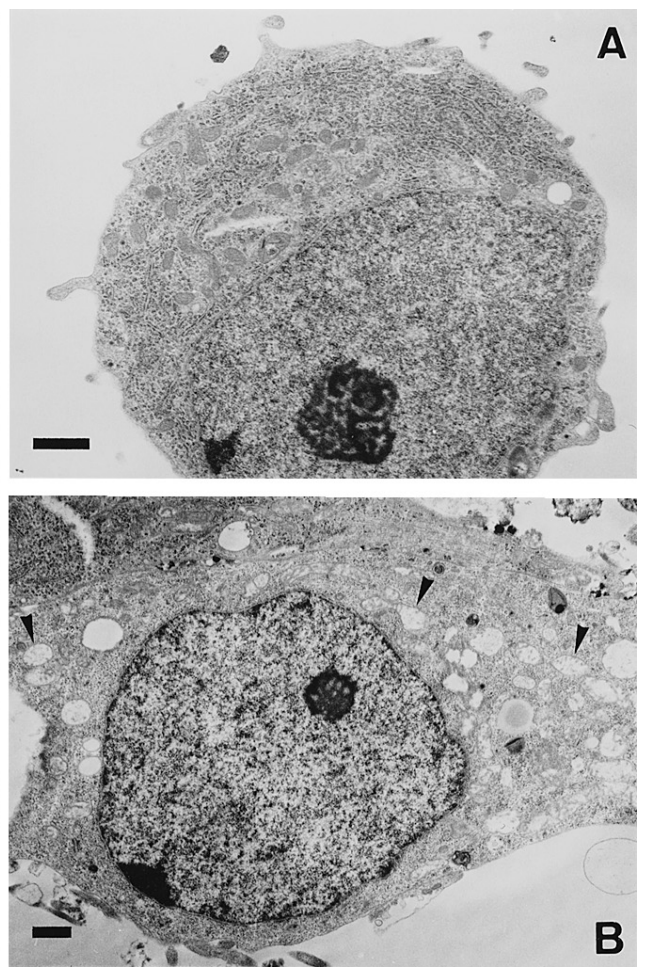

Fig. 2. Morphological Features of 5-HT-Induced Death of CCCs

At 6 DIV, 5-HT (0.1 mM) was added to cultures and cells were fixed at 9 DIV and then morphologically examined as described in Materials and Methods. I: Fluorescence microscope images of nuclei stained with the DNA-specific fluorochrome Hoechst dye. A, Untreated control cells. B, Cells exposed to 5-HT. Note that cells treated with 5-HT did not exhibit any nuclear fragmentation and/or chromatin condensation. Scale bar, $20 \mu \mathrm{m}$. II: Electron micrographs showing representative CCCs in an untreated control culture (A), and a culture exposed to 5-HT (B). Note the high degree of vacuolization in the cytoplasm and damaged inner mitochondrial membranes (arrowheads) in cell in B. Scale bar, $1 \mu$ m.

\section{Surviving Cells (\% of 6 DIV)}

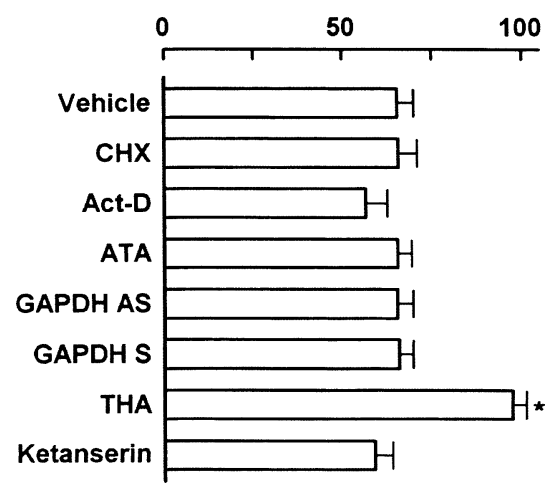

Fig. 3. 5-HT-Induced Neuronal Death Is Not Protected by the Addition of Classical Apoptotic Inhibitors and GAPDH Antisense Oligonucleotide

CCCs were exposed to $0.1 \mathrm{~mm} 5$-HT and the neuronal survival was assessed as described in the legend to Fig. 1. Cultures were pretreated with CHX $(0.3 \mu \mathrm{g} / \mathrm{ml})$, Act-D $(0.015 \mu \mathrm{g} / \mathrm{ml})$, ATA $(10 \mu \mathrm{M})$, GAPDH oligonucleotides $(10 \mu \mathrm{M})$, THA $(10 \mu \mathrm{M})$, or ketanserin $(10 \mu \mathrm{M})$. All reagents were added $1 \mathrm{~h}$ before exposure to 5 -HT. Values are means \pm S.E.M. of three independent experiments. $* p<0.01$ compared with the untreated (vehicle, i.e., $\mathrm{H}_{2} \mathrm{O}$ ) control, using Student's $t$ test. Bars: GAPDH AS, plus GAPDH antisense oligonucleotide; GAPDH S, plus GAPDH sense oligonucleotide.

trations of 5-HT induced cytotoxicity in primary cerebrocortical neuronal cultures for the first time. This neurotoxicity occurred substantially with 5-HT at $100 \mu \mathrm{M}$ (Fig. 1). So far, the mechanism(s) underlying 5-HT-exposed neuronal death is unclear. However, it seems unlikely that 5-HT neurotoxicity is merely due to abnormalities in the signaling pathway via its and/or another concerted receptor(s) because certain antagonists specific to 5-HT or NMDA receptor did not affect
A

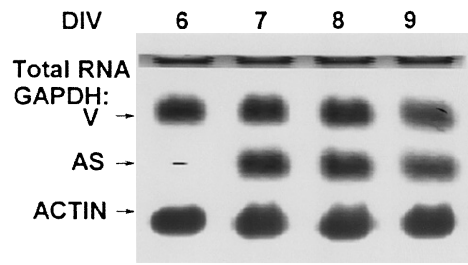

B

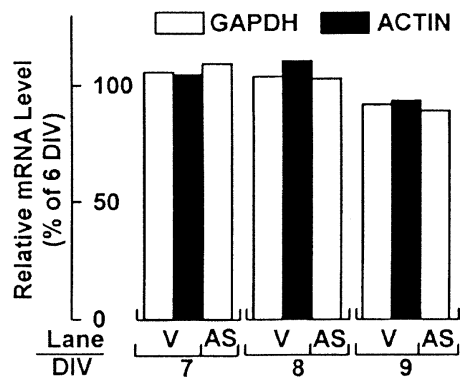

\% Surviving cells $93.2 \quad 94.0 \quad 83.0 \quad 81.5 \quad 65.5 \quad 65.0$ exposed to $5-\mathrm{HT}$

Fig. 4. Northern Blot Analysis of GAPDH and $\beta$-Actin mRNA Levels in CCCs during Exposure to 5-HT: Time Course and Non-effect of GAPDH Antisense Oligonucleotide (A) and Levels of Quantified GAPDH and $\beta$ Actin (ACTIN) mRNAs in Bar Graphs and the Assessment of Surviving Cells During Exposure to 5-HT (B)

CCCs were pretreated with the antisense oligonucleotide and then exposed to 5-HT as described in the legend to Fig. 3. Experimental conditions for Northern blotting and the assessment of surviving cells are as described in Materials and Methods. Levels of those mRNAs and surviving cells at 7-9 DIV are expressed as values relative to the 6 DIV control. Results are from a typical experiment; virtually identical results were obtained from two independent experiments. Note the relatively constant level of GAPDH mRNA at 7-8 DIV and slight decrease in its level at 9 DIV to reflect on the maximum decrease in surviving cells in this paradigm, corresponding with levels of ACTIN mRNA. Lanes: V, plus vehicle; AS, plus GAPDH antisense oligonucleotide. 
this toxicity (Fig. 3). Therefore, it is conceivable that several detrimental effects of 5-HT, including association with the production of free radicals, may be implicated in the cell death. ${ }^{16)}$ Morphological and biochemical investigations distinctly revealed that the 5-HT-exposed neurons resulted in necrotic cell death (Figs. 2, 3). Among the tested agents, only THA furnished a neuroprotective effect in this paradigm. In this context, we have reported that THA $(3-10 \mu \mathrm{M})$ delays age-induced apoptosis in the same neuronal cultures, by suppressing the overexpression of GAPDH. ${ }^{17)}$ However, it is well documented that THA has diverse functions such as blockade of 4-aminopyridine-sensitive potassium channels and promoting the muscarinic receptor-coupled phosphoinositide turnover. ${ }^{18,19)}$ It is therefore not surprising that THA protects against not only apoptosis but also necrosis.

We have reported that overexpression of GAPDH is involved in neuronal apoptosis induced by several stimuli where GAPDH antisense oligonucleotides specifically block the increment (2- to 3-fold) of GAPDH mRNA levels occurring prior to neuronal death; however, these do not affect the basal, constitutive mRNA levels. ${ }^{5,9,20)}$ This suggests that there may be multiple pools of GAPDH mRNA (and protein) that are subjected to differential regulation. Concerning this proposal, we have recently demonstrated that overexpression of GAPDH and its subsequent nuclear accumulation robustly induces apoptotic death in the COS-7 cells transfected with cloned GAPDH cDNAs. ${ }^{21)}$ Our findings in the present study evidenced the specific implication of GAPDH only in apoptosis for the following reasons: 1) in the 5-HT-exposed necrotic paradigm, any enhancement of GAPDH mRNA level prior to cell death was undetected, and its expression degree in the death pathway was parallel to that of $\beta$-actin, a normalizer (Fig. 4); and 2) GAPDH antisense transfection studies certainly supported the irrelevance of GAPDH overexpression to the necrotic death process (Figs. 3, 4).

Finally, mounting evidence indicates that pro-apoptotic protein GAPDH is an important putative molecular target to develop therapeutic agents for the treatment of certain forms of neurodegenerative disorders. The first such efforts have been reported recently. ${ }^{22-25)}$

Acknowledgements We are indebted to Drs. Ryoichi Ishitani and Katsumi Tsuchiya (Josai University) for review- ing the manuscript.

\section{RERFERENCES}

1) Oppenheim R. W., Annu. Rev. Neurosci., 14, 453-501 (1991).

2) Wyllie A. H., Kerr J. F. R., Currie A. R., Int. Rev. Cytol., 68, 251-306 (1980).

3) Thompson C. B., Science, 267, 1456-1462 (1995).

4) Hardy J., Gwinn-Hardy K., Science, 282, 1075-1079 (1998).

5) Ishitani R., Sunaga K., Hirano A., Saunders P., Katsube N., Chuang D.-M., J. Neurochem., 66, 928-935 (1996).

6) Ishitani R., Kimura M., Sunaga K., Katsube N., Tanaka M., Chuang D.-M., J. Pharmacol. Exp. Ther, 278, 447-454 (1996).

7) Sawa A., Khan A. A., Hester L. D., Snyder S. H., Proc. Natl. Acad. Sci. U.S.A., 94, 11669-11674 (1997).

8) Ishitani R., Tanaka M., Sunaga K., Katsube N., Chuang D.-M., Mol. Pharmacol., 53, 701-707 (1998).

9) Ishitani R., Sunaga K., Tanaka M., Aisita H., Chuang D.-M., Mol. Pharmacol., 51, 542-550 (1997).

10) Burke J. R., Enghild J. J., Martin M. E., Jou Y.-S., Myers R. M., Roses A. D., Vance J. M., Strittmatter W. J., Nat. Med., 2, 347-350 (1996).

11) Schulze H., Schuler A., Stüber D., Döbeli H., Langen H., Huber G., J. Neurochem., 60, 1915-1922 (1993).

12) Sunaga K., Chuang D.-M., Ishitani R., Neurosci. Lett., 163, 27-30 (1993).

13) Schramm M., Eimerl S., Costa E., Proc. Natl. Acad. Sci. U.S.A., 87, 1193-1197 (1990).

14) Joseph R., D’Andrea G., Oster S. B., Welch K. M. A., Stroke, 20, 38 44 (1989).

15) Harper A. M., Mackenzie E. T., J. Physiol., 271, 721-733 (1977).

16) Joseph R., Tsering C., Grunfeld S., Welch K. M. A., Neurosci. Lett., 136, 15-18 (1992).

17) Katsube N., Sunaga K., Aishita H., Chuang D.-M., Ishitani R., J. Pharmacol. Exp. Ther., 288, 6-13 (1999).

18) Hershkowitz N., Rogawski M. A., Mol. Pharmacol., 39, 592-598 (1991).

19) Sunaga K., Chuang D.-M., Ishitani R., J. Pharmacol. Exp. Ther, 264, 463-468 (1993).

20) Ishitani R., Chuang D.-M., Proc. Natl. Acad. Sci. U.S.A., 93, 99379941 (1996).

21) Tajima H., Tsuchiya K., Yamada M., Kondo K., Katsube N., Ishitani R., Neuro Report, 10, 2029-2033 (1999).

22) Kragten E., lalande I., Zimmermann K., Roggo S., Schindler P., Müller D., van Oostrum J., Waldmeier P., Fürst P., J. Biol. Chem., 273, 58215828 (1998).

23) Carlile G. W., Chalmers-Redman R. M. E., Tatton N. A., Pong A., Borden K. L. B., Tatton W. G., Mol. Pharmacol., 57, 2-12 (2000).

24) Katsube N., Ishitani R., CNS Drug Rev., 6, 21-34 (2000).

25) Ishitani R., Tajima H., Takata H., Tsuchiya K., Kuwae T., Yamada M., Takahashi H., Tatton N. A., Katsube N., Prog. Neuropsychopharmacol. Biol. Psychiatry, 27, 291-301 (2003). 